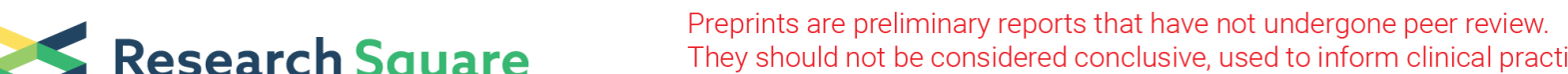 \\ They should not be considered conclusive, used to inform clinical practice, or referenced by the media as validated information.
}

\section{An Adaptive Design To Screen, Treat And Retain People With Opioid Use Disorders Who Use Methamphetamine In Methadone Clinics (STAR- OM) Study Protocol of A Clinical Trial}

\section{Giang Le Minh}

Hanoi Medical University

Trang Nguyen Thu ( $\nabla$ trangthu@hmu.edu.vn )

Hanoi Medical University https://orcid.org/0000-0003-3261-2098

Diep Nguyen Bich

Hanoi Medical University

Thuy Dao Thi Dieu

Hanoi Medical University

Thuy Dinh Thanh

Hanoi Medical University

Hoe Han Dinh

Hanoi Medical University

Van Hoang Thi Hai

Hanoi Medical University

Truc Thai Thanh

University of Medicine and Pharmacy at Ho Chi Minh City

Hoa Hong Nguyen

University of Medicine and Pharmacy at Ho Chi Minh City

Lai Nguyen Ly

University of Medicine and Pharmacy at Ho Chi Minh City

Linh Pham Thi Dan

University of Medicine and Pharmacy at Ho Chi Minh City

Vi Vu Thi Tuong

University of Medicine and Pharmacy at Ho Chi Minh City

Cathy J. Reback

University of California

Arleen Leibowitz

University of California

Li Li

University of California 


\section{Chunqing Lin}

University of California

\section{Michael Li}

University of California

\section{Dung Do Van}

University of Medicine and Pharmacy at Ho Chi Minh City

\section{Steve Shoptaw}

University of California

\section{Research Article}

Keywords: Methamphetamine, Amphetamine-type stimulants, methadone, opioid substitution, Vietnam

Posted Date: February 7th, 2022

DOI: https://doi.org/10.21203/rs.3.rs-1002929/v1

License: (c) (i) This work is licensed under a Creative Commons Attribution 4.0 International License.

Read Full License 


\section{Abstract}

Background: Methamphetamine use could jeopardize the current efforts to address opioid use disorder and HIV infection. Evidence-based behavioral interventions (EBI) are effective in reducing methamphetamine use. However, evidence on optimal combinations of EBI is limited. This protocol presents a Type- 1 effectiveness-implementation hybrid design to evaluate the effectiveness, costeffectiveness of adaptive methamphetamine use interventions and their implementation barriers in Vietnam.

Method: Design: Participants will be first randomized into two frontline interventions for 12 weeks. They will then be placed or randomized to three adaptive strategies for another 12 weeks. An economic evaluation and an ethnographic evaluation will be conducted alongside the interventions. Participants: We will recruit 600 participants in 20 methadone clinics. Eligibility criteria: 1) age 16+, 2) Alcohol, Smoking and Substance Involvement Screening Test (ASSIST) scores 10 or more for methamphetamine use or confirmed methamphetamine use with urine drug screening; 3) willing to provide three pieces of contact information; 4) having a cell phone.

Outcomes. Outcomes are measured at 13-, 26- and 49-week and throughout the interventions. Primary outcomes include: (1) increase in HIV viral suppression; (2) reduction in HIV risk behaviors; and (3) reduction in methamphetamine use.

COVID-19 response: We developed a response plan for interruptions caused by COVID-19 lockdowns to ensure data quality and intervention fidelity.

Discussion: This study will provide important evidence for scale-up of EBls for methamphetamine use among methadone patients in limited-resource settings. As the EBIs will be delivered by methadone providers, they can be readily implemented if the trial demonstrates effectiveness and cost-effectiveness.

Trial registration: NCT04706624. Registered 13 January 2021.

https://clinicaltrials.gov/ct2/show/NCT04706624

\section{Introduction}

The global rise of methamphetamine use could jeopardize current intervention efforts to address the twin epidemics of opioid use disorder (OUD) and HIV infection. Use of methamphetamine is increasingly common among people with primary OUD (1-3). Prevalence of methamphetamine use disorders is increasing in Vietnam (4), raising concerns about increased risk of HIV infection (5-8) and disruption of the substance use treatment systems, especially methadone programs $(9,10)$. Methamphetamine use among people living with HIV could decrease retention in care, hinder medication adherence, accelerate viral replication, and further HIV disease progression (11-15). Other countries beyond South-East Asia encounter similar challenges (16-18). In low-and-middle-income countries, it is vital to identify costeffective models of adapted evidence-based practices for addressing substance use disorders $(19,20)$. 
Although there are no approved pharmacological treatments for methamphetamine use, evidence-based behavioral interventions (EBI) such as motivational interviewing, contingency management, cognitive behavioral therapy, including Matrix model have shown efficacy in reducing methamphetamine use (2123). However, we need to identify optimal combinations of EBI for effectiveness and cost-effectiveness as many people in treatment face challenges to retention and sustained reductions in use.

Motivational Interviewing. Motivational interviewing helps individuals to evaluate the pros and cons to change drug use and to develop personalized change behaviors. Motivational interviewing can be used in a single session or in multiple sessions (22). Polcin et al. (2014) compared two motivational interviewing conditions ( 9 sessions vs. 1 session) and found that both groups showed significant reductions in methamphetamine use without differences between the two groups (24). A greater reduction in psychiatric symptoms including anxiety and depression was found among those receiving more motivational interviewing sessions $(21,24,25)$.

Contingency Management. Contingency management has shown the strongest evidence in treating methamphetamine use disorders $(21,26-30)$. It is also effective in reducing other drug use including alcohol, cannabis, nicotine and opioids (31). Contingency management is based on the theory of operant conditioning where incentives are used to strengthen the target behavior such as abstinence, reduction of sexual risk behaviors or other health-promoting behaviors like retention or adherence to treatment (27). Contingency management effects are enhanced in combination with other psychosocial interventions or education (22). A recent meta-analysis shows contingency management is more efficacious than other EBI up to one year following the discontinuation of reinforcers (32).

Matrix Model. The Matrix model has shown greater reduction in methamphetamine use, risky behaviors and more days of abstinence compared to non-standardized outpatient treatment approaches $(21,23)$. This intervention combines different elements of effective approaches including cognitive and behavioral treatment using accurate information on the effects of stimulants, relapse prevention skills training, 12-step program participation and family education (33). Its manualized treatment protocol ensures fidelity when the model is implemented in different settings.

SMS Text Messages. Using SMS text messages with people who use methamphetamine has been shown to reduce methamphetamine use and HIV-related sexual transmission behaviors $(34,35)$, and increase retention in HIV care among some key populations (36). Scripted unidirectional texts outperform bidirectional interactive text-messaging conversations in reducing methamphetamine use and HIV sexual risk behaviors, and are more cost-effective than in-person therapies (37). Theory-driven messaging might better benefit people in the early stages of behavior change (e.g. non-treatment seeking participants) than people who are already seeking help (34).

Despite some demonstrated efficacy, few studies have shown ways to optimize and combine treatment approaches for methamphetamine use disorders. Qualitative reports show patients found contingency management beneficial when combined with motivational interviewing and cognitive behavioral techniques for methamphetamine use disorders (38). Combined motivational interviewing and cognitive 
behavioral treatment show efficacy in reducing methamphetamine use in HIV-positive MSM (39). Evidence supports combining psychosocial treatment with medication-assisted treatment in people with OUD (40), but it is unclear whether patients with comorbid methamphetamine use disorder will experience similar benefits.

Integrating screening and brief interventions (41-43), contingency management or conditional cash transfer $(6,44,45)$, and cognitive behavioral therapy $(46-48)$ for the management of substance use disorders requires trained health professionals. This is challenging in settings where human resource for mental health/substance use is scarce. Therefore, besides identifying optimal combination of EBI, it is essential to recognize potential barriers to the implementation of these strategies. Our study named 'Screen, Treat and Retain people with opioid use disorders who use methamphetamine in methadone clinics'(STAR-OM) proposes to explore these questions. The study has three aims:

Aim 1: To develop and to compare the effectiveness of two frontline interventions and four adaptive strategies in improving HIV and substance use outcomes among people with OUD who use methamphetamine at methadone clinics in the two largest cities in Vietnam.

Aim 2: To compare cost-effectiveness of two frontline interventions and of four adaptive strategies in improving both HIV and substance use outcomes among people with OUD who use methamphetamine at methadone clinics.

Aim 3: To identify the structural, provider, and patient-level factors that influence adoption and scale-up of the studied model in methadone clinics.

\section{Method}

\section{Overview of Study Design}

The study deploys a Type-1 effectiveness-implementation hybrid design to evaluate the effectiveness of the proposed adaptive interventions and gather data on the implementation (49). To evaluate the effectiveness of the interventions, the study employs a Sequential Multiple Assignment Randomized Trial (SMART) design. In the first phase, participants will be randomized into two frontline interventions for 12 weeks. Based on their outcome at the end of this phase, they will be placed or randomized into three adaptive strategies for another 12 weeks (Figure 1). The economic evaluation that addresses Aim 2, aims to weigh public health and societal costs against public health and societal benefits attributed to the interventions of different intensities with a time horizon of 12 months. To address Aim 3, we will conduct an ethnographic evaluation to identify the multi-level factors that influence the adoption and scale-up of the interventions in methadone clinics. The ethnographic evaluation is guided by the Consolidated Framework for Implementation Research (CFIR) (50,51). The CFIR assesses five domains of interventions, outer settings, inner settings, provider characteristics and participant characteristics. The evaluation includes pre- and post-intervention in-depth interviews with key informants who participate in the study 
and ethnographic observation with participants in their daily activities at the clinics and in the community settings.

\section{Settings}

Participants are recruited from the methadone clinics in Hanoi and Ho Chi Minh City (HCMC) - the two largest urban settings in Vietnam. As of March 2021, there are 23 methadone clinics in HCMC and 18 in Hanoi, treating 5047 and 4655 patients, respectively $(52,53)$. Criteria for selecting clinics include number of patients, estimated prevalence of methamphetamine use, availability of human resources to implement study interventions, and space for intervention activities. 10 clinics in each city will be randomly selected from those that meet the criteria.

\section{Study schedule}

The study pilot phase started in November 2020. The full study implementation phase began in May 2021. To recruit 200 HIV-positive and 400 HIV-negative methamphetamine-using methadone participants from both cities, we will screen about 5,000 patients in 20 methadone clinics. A cluster of four methadone clinics will start every six months. We expect to complete the last cluster in October 2023.

\section{Participants}

\subsection{Participants under methadone treatment}

We will recruit 600 methadone participants with the following eligibility criteria: 1) age 16 or older, 2) Alcohol, Smoking and Substance Involvement Screening Test (ASSIST) scores 10 or more for methamphetamine use or confirmed methamphetamine use with urine drug screening (UDS); 3 ) willing to provide at least three pieces of contact information; 4) has a cell phone that is capable of receiving text messages. The criteria of ASSIST scores and UDS had been modified after the pilot implementation (see Section 14.2.1). Exclusion criteria are: 1) psychosis or other interfering problems; 2) inability to understand study procedures by research assistants' judgment. While World Health Organisation recommends the ASSIST score of 4 or more, regardless of UDS, as a cut-off point for interventions, drawing from the results of the pilot phase we have chosen the cut-off point of ASSIST score 10 or more to ensure a sufficient number of participants move into the adaptive phase.

\subsection{Methadone providers}

Methadone providers will conduct the study interventions under the clinical supervision of the study master counselors. All methadone providers have been trained on basic addiction medicine and will receive further training to deliver the study interventions. We will conduct in-depth interviews with them before and after the intervention phase.

\section{Randomization and Interventions}


An investigator will stratify participants by HIV status and randomize them into two frontline interventions using REDCap, as shown in Figure 1. She will send the allocation results to the site research assistants who will then inform providers at the study clinics. Participants will receive two individual sessions of motivational interviewing with their counselors before they get into the frontline interventions and, in Week 13, before the adaptive strategies start. These sessions would boost participants' motivation for intervention and provide them with greater details of the upcoming intervention activities.

\subsection{Two frontline interventions (Weeks 1-12)}

- High intensity: Participants in this arm receive contingency management with the escalating-andreset schedule throughout 12 weeks (54). This schedule means participants receive increasing rewards for consecutive negative UDS but if the streak of negative UDS is broken, their rewards return to the starting level. The maximal reward value over 12 weeks is $\$ 150$ USD, equivalent to the average monthly income of our participants.

- Low intensity: Participants in this arm receive contingency management for the first six weeks with the maximal reward value of $\$ 40$ USD. For the last six weeks, they attend weekly group education sessions. The topics of group education include 1) Addiction mechanism, 2) Road to recovery, 3) Coping with triggers, 4) Boredom, 5) Building trust and 6) Relapse prevention.

Participants with four consecutive UDS negative with methamphetamine in weeks 11 and 12 are considered to be responsive to frontline interventions. Others are considered non-responsive. Responders to frontline interventions are placed in the maintenance treatment arm. Non-responders are randomized to either enhanced treatment 1 or 2.

\subsection{Three adaptive strategies (Weeks 14-25)}

- Maintenance treatment: Participants receive two daily automatic unidirectional scripted SMS reminders plus one weekly self-monitoring assessment message over 12 weeks.

- Enhanced treatment 1: Participants attend 12 weekly Matrix group counseling sessions facilitated by the clinic counselors. Our Matrix intervention has the same structure, albeit it is shorter than the original 24-sessions model (33).

- Enhanced treatment 2: Participants receive the same Matrix intervention plus contingency management over 12 weeks.

\section{Economic evaluation}

\subsection{Definition of costs and data collection}

We will conduct activity-based costing for each intervention arm over the 20 methadone clinics. A template developed by UNAIDS is adapted to collect data on salaries for personnel and consultants, physical resources, clinical supplies and miscellaneous charges necessary to deliver each intervention 
type (55). Furthermore, any out-of-pocket or indirect costs to the participant will be collected at baseline, and at 13- and 26-week.

\subsection{Definition of effectiveness measures}

Measures of cost-effectiveness analysis corresponded to the outcomes of interest in Aim 1 including (1) Substance use, (2) HIV risk behaviors among HIV-negative participants and HIV viral load, HIV adherence among HIV-positive participants and (3) Quality of life (Table 1). The cost-effectiveness analysis will measure the increment in cost between contrasted interventions divided by the increment in effectiveness measures.

\section{Ethnographic evaluation}

\subsection{Pre-post intervention in-depth interviews}

In each cluster, we will interview 12 key informants including methadone providers, clinic managers and participants under methadone treatment participating in the study. We will select at minimum 6 participants under treatment so that include old and young, employed and not employed, and both responsive and non-responsive participants. All participants will receive VND 200000 ( \$10 USD) for their time in each interview. Interviews will be audio-recorded and transcribed verbatim.

\subsection{Ethnographic observation}

This activity is composed of two elements. The first element involves ethnographers spending time with participants with their consent in intervention sessions and other daily activities in the clinic. Such observations will build a rich picture of interventions and intervention settings, including interactions between various groups (intervention providers, other staff, and participants). The second element involves the study master counselors to observe random intervention sessions and assess the fidelity of intervention delivery using a checklist.

\section{Training and fidelity monitoring}

In each selected clinic, a physician, two counselors and one nurse will participate in the study as intervention providers. The physician will ensure referral to HIV and psychiatric services when necessary; two counselors will run motivational interviewing, group education sessions and Matrix meetings; the nurse will collect urine twice a week and conduct contingency management based on the UDS results. Before the start of the intervention, to ensure the accuracy, integrity and fidelity to the EBIs, all intervention staff at methadone clinics will (1) receive didactic training on the theory behind the approach; (2) evaluate their comprehension of the concepts within and behind the approach; (3) watch a video of a Master Behavioral Counselor conducting intervention sessions and discuss the details of the session, and (4) conduct at least two pilot intervention instances. All intervention sessions, except contingency management, will be audio recorded, transcribed, and coded to ensure intervention fidelity. Intervention 
staff who have lower levels of intervention integrity or who have significant drift will be provided detailed feedback and supervision until there is parity with other staff.

\section{Outcome measures}

Outcome measures will be assessed at 13-, 26- and 49 weeks after the first week of frontline interventions and throughout the interventions.

Primary outcomes include: (1) increase in HIV viral suppression for HIV-positive participants at 26- and 49-weeks; (2) reduction in HIV risk behaviors for both HIV-positive and HIV-negative participants at 26and 49-weeks; and (3) reduction in methamphetamine use at 13-, 26- and 49-weeks measured by UDS (point abstinence) and self-report (continuous abstinence and longest period).

Secondary outcomes include: (1) adherence to antiretroviral treatment for HIV-positive participants; 2) frequency of HIV testing for HIV-negative participants; 3) heroin use with UDS; 4) opioid overdose; and, 5) quality of life.

\section{Sample size determination}

Sample sizes were chosen to compare primary outcomes based on first-stage randomization into one of two groups: high intensity or low intensity frontline interventions. Sample size calculations are conducted in PASS 2008 (56) for a two-group comparison of binary outcomes, a power of $80 \%$, a $5 \%$ alpha level, and a conservative attrition rate of $20 \%$. Using estimates from our prior work, we anticipate base rates of $80 \%$ to $90 \%$ for substance use and $60 \%$ to $70 \%$ for viral suppression. Based on these assumptions and a proposed sample of 200 HIV-positive participants (with 100 participants per group), we can detect randomization group differences of $20 \%$ or more for binary outcomes, such as substance use and viral load suppression. We can detect even smaller group differences for substance use outcomes in the proposed sample of 400 HIV-negative participants and the combined sample of HIV-positive and negative participants. If estimated outcome probabilities are similar between first-stage randomization groups at 12 weeks, we will pool 12-week results for even greater power in evaluating second-stage randomization differences.

\section{Data management}

Different datasets collected from different sources will be linked through a unique identification code using REDCap (Research Electronic Data Capture) for quantitative data. Data will be uploaded in real-time from the 20 study clinics onto our database. The study data manager will assess transferred data for completeness, query sites regarding any inconsistencies, and code merged data files for analysis.

For qualitative data, field notes written on site are expanded and recorded electronically within 24 hours. After removing all personal identifiable information, the research team will upload password-protected 
transcripts on a secured database. The transcripts will be uploaded into Atlas.ti software to organize data and facilitate analysis.

\section{Data analysis}

\subsection{To assess effectiveness}

For Aim 1, adaptive intervention strategies will be evaluated through regression models. Models will include two indicator variables representing: 1) the first randomization to either frontline intervention; and 2) the second randomization of non-responders to either Matrix only or Matrix+contingency management at 12-week. An interaction term of these two indicators will account for any additional impact of the second stage randomization among non-responders at 49-week. The models will adjust for within-person correlations (57) as well as individual demographic characteristics and clinic characteristics. A weighting and replication approach will be used to adjust for the way that randomization is allocated; responders are only randomized once and non-responders are randomized twice $(58,59)$. Predicted probabilities of viral suppression and substance use at 49-week across first and second stage intervention options will be estimated from logistic models.

\subsection{To assess cost-effectiveness}

We will calculate Cost Effectiveness Ratios (CER) for each of the intermediate and final outcomes. The CER is in the broadest terms the difference in per capita costs of administering one intervention (C1-C2) relative to a second, divided by the difference in outcomes between the two interventions (01-02):

$$
C E R=\frac{C 1-C 2}{O 1-O 2}
$$

For example, calculating the CER for adding contingency management to Matrix for non-responders would yield a CER equation. In calculating the CER of high vs. low intensity contingency management at the first randomization stage, the entire range of subsequent costs will be included. Costs of delivering the interventions will be derived from clinic records of time and other inputs, as well as incentive payments, thus providing an estimate of CER from the medical system perspective. We will also evaluate CERs from a societal perspective, using a broad definition of costs, including the social costs of incarceration.

We will conduct sensitivity analyses (60) to estimate the extent to which the CER calculation is affected by differences in assumptions about the size of the differences in intervention effect. In particular, we will determine how sensitive the CER is to assumptions that the difference in treatment effect is one standard deviation below or above the mean estimated effect size. Similarly, we will estimate the sensitivity of conclusions to costs that are one standard deviation below or above the estimated mean. 
The qualitative analysis team will read and provide a narrative summary for each transcript. A codebook will be developed based on these summaries. Memo-writing and code-refining will be conducted throughout the analysis. Iterative analyses assess convergence of patient, provider and organizational dimensions on study measures as well as the context of the policy subsystems, cross system interactions, and resource allocation.

\section{Ethics}

Prior to participation in the trial, the participant will be informed about the research. Participants will complete a short questionnaire about the study objectives and main activities to show how they understand the study. Research assistants will provide more explanation based on the results of the questionnaire. If participants agree to join the study, they will sign a consent form. Each participant will be assigned a unique identifier at the time of screening.

\section{Challenges and adaptations}

\subsection{Intervention adaptation}

Between July and October 2020, we conducted 4 focus group discussions (FGD) of a convenience sample of participants from four methadone clinics in the downtown and suburbs of Hanoi and HCMC to inform intervention content and refinement. Respondents reported information on local taxonomy and patterns of methamphetamine use, triggering situations, methamphetamine-related sexual risks, motivations for seeking treatment, and perceived acceptability of the adaptive interventions.

The pilot implementation lasted 12 weeks from November 2020 through February 2021. It identified issues to be addressed before the full implementation. At the conclusion of the pilot, we conducted 2 FGD with patients and 1 FGD with providers participating in the pilot to gauge their feedback about the interventions.

\subsection{Challenges and modifications}

\subsubsection{Modification of eligibility criteria}

With the cut-off point of ASSIST $\geq 4$ and methamphetamine-positive UDS as originally proposed, there were 26 and 52 eligible participants in two pilot clinics in Hanoi and HCMC, respectively (see Table 2). For the pilot implementation, we randomly recruited 42 participants with ASSIST score $\geq 4$ or methamphetamine-positive UDS. After the frontline intervention, 16 (38\%) participants were nonresponders and randomized into adaptive interventions. At least $50 \%$ of the original sample must transition to the adaptive phase for sufficient statistical power. Thus, we decided to recruit more participants with severe use of methamphetamine, as evidenced in both ASSIST score $\geq 10$ and methamphetamine-positive UDS. Furthermore, to recruit enough participants for the frontline intervention phase, given most other clinics are smaller than the two pilot ones, we decided to use ASSIST score 'OR' UDS instead of 'AND' to increase the pool of potential participants. We kept the criterion of 
methamphetamine-positive UDS to compensate for participants with lower ASSIST scores due to desirability bias.

\subsubsection{Modification to minimize the impacts of the COVID-19 pandemic}

Since late April 2021, the COVID-19 epidemic in Vietnam was severe. HCMC applied a strict lockdown from early July 2021 through September 2021. Some methadone clinics were temporarily closed due to confirmed COVID-19 cases among patients, medications were delivered at community-based healthcare centers. In the clinics that remained open, clinical activities other than medication dispensing ceased in order to minimize contact between providers and patients. Unlike HCMC, clinical activities in Hanoi continued, albeit at levels lower than pre-pandemic. In addition, methadone clinics in both cities suffered from staff shortages as many staff were deployed to support ongoing COVID-19 prevention and treatment activities. With advice of the study's Scientific Advisory Board, we developed a response plan to potential COVID-19 interruptions to minimize the pandemic's impact (see Figure 2).

\section{For intervention:}

- For group education and Matrix sessions, we will conduct small groups of five or fewer people during the surge of the COVID-19 pandemic when the city authorities forbid large meetings. If we cannot conduct group sessions, we will provide individual sessions of the same content to ensure all participants receive interventions.

- For participants who miss scheduled visits due to COVID-19, we will consider whether to resume the intervention where they left off or to restart their intervention phase. This will depend upon: 1) the length of the interruption (15 days or less); and, 2) whether participants have gone through $50 \%$ of their scheduled intervention sessions before the interruption.

\section{Discussion}

The STAR-OM study is among the first studies to evaluate different combinations of EBIs for methamphetamine use among methadone patients in low-and-middle-income countries. The study will provide effectiveness and cost-effectiveness evidence for scaling up these interventions. The SMART design assesses different treatment strategies for participants who respond differently to frontline interventions. The combination of trial and ethnographical study will provide insights on factors at multiple levels that need to be considered in decision-making. The adaptation and pilot implementation of EBIs will make them culturally sound to local participants. As the interventions will be delivered by methadone providers at methadone clinics, they can be readily implemented if the trial demonstrates they help.

The participation of some participants can be interrupted due to drug-related police arrest or methadone treatment fatigue. This limitation can be minimized as we will select clinics with low drop-out rates. We have officially informed the local police on the study implementation and received approval from both national and local authorities. While this measure does not prevent participants from being arrested, 
especially when they are involved in illegal activities, it could reduce attrition. Furthermore, the COVID-19 pandemic and containment measures could pose challenges for the study implementation. With the response plan developed for potential interruption scenarios, we believe the study will be implemented safely and will maintain a high-level of data quality and intervention fidelity.

\section{Conclusion}

\section{TRIAL STATUS}

Protocol version 2.0, dated 25 March 2021

Recruitment start date: 14 June 2021

Estimate recruitment completion date: 31 March 2024

Ethics approval and consent to participate: Ethics approval has been obtained through Hanoi Medical University (\#122/GCNHDDDNCYSH-ĐHYHN) and University of Medicine and Pharmacy in HCMC, Vietnam (\#433/HDDD-ĐHYD).

Consent for publication: Not applicable

Availability of data and materials: Not applicable

Competing interests: The authors declare that they have no competing interests

Funding: The study is funded by the National Institute on Drug Abuse (1R01DA050486-01). This funding source had no role in the design of this study and will not have any role during its execution, analyses, interpretation of the data, or decision to submit results.

Authors' contributions: LMG, DVD and SS designed and lead the study. NTT oversees the implementation in the two study sites and led the writing of the manuscript. NBD analyzed the study pilot data. DTDT and AL wrote the section on economic evaluation. HDH, NLL and PTDL revised the intervention strategies with inputs from the pilot implementation. TTT, HHN, CR, CL and ML contributed to the revision of the manuscript. All authors read and approved the final manuscript.

Acknowledgments: We thank our research assistants for their help in data collection. We are grateful to the methadone practitioners and patients for their participation in our study. The study's advisory board provided us with valuable advices on how to deal with the possible implementation interruptions due to COVID-19 pandemic.

\section{Declarations}

TRIAL STATUS 
Protocol version 2.0, dated 25 March 2021

Recruitment start date: 14 June 2021

Estimate recruitment completion date: 31 March 2024

Ethics approval and consent to participate: Ethics approval has been obtained through Hanoi Medical University (\#122/GCNHDDDNCYSH-ĐHYHN) and University of Medicine and Pharmacy in HCMC, Vietnam (\#433/HDDD-ĐHYD).

Consent for publication: Not applicable

Availability of data and materials: Not applicable

Competing interests: The authors declare that they have no competing interests

Funding: The study is funded by the National Institute on Drug Abuse (1R01DA050486-01). This funding source had no role in the design of this study and will not have any role during its execution, analyses, interpretation of the data, or decision to submit results.

Authors' contributions: LMG, DVD and SS designed and lead the study. NTT oversees the implementation in the two study sites and led the writing of the manuscript. NBD analyzed the study pilot data. DTDT and AL wrote the section on economic evaluation. HDH, NLL and PTDL revised the intervention strategies with inputs from the pilot implementation. TTT, HHN, CR, CL and ML contributed to the revision of the manuscript. All authors read and approved the final manuscript.

Acknowledgments: We thank our research assistants for their help in data collection. We are grateful to the methadone practitioners and patients for their participation in our study. The study's advisory board provided us with valuable advices on how to deal with the possible implementation interruptions due to COVID-19 pandemic.

\section{Organisational structure and responsibilities}

\section{Principal Investigators: Le Minh Giang and Steve Shoptaw}

Design and conduct of STAR-OM

Preparation of protocol and revisions

Organising scientific advisory board meetings

Publication of study reports

\section{Co-Investigators: Hoang Thi Hai Van, Nguyen Van Tuan, Do Van Dung, Thai Thanh Truc}

Development of the study standards of operation and data collection tools

Supervising the data collection and analysis process

Development of manuscripts 
Steering committee: Gavin Bart, Don Desjarlais, Khuat Thi Hai Oanh, Hoang Dinh Canh, Richard Rawson Agreement of final protocol

Reviewing progress of study and if necessary agreeing changes to the protocol to facilitate the smooth running of the study.

\section{Data Manager: Nguyen Bich Diep}

Maintenance of trial IT system and data entry

Data verification and analysis

\section{Project Director: Nguyen Thu Trang}

Overseeing the study implementation

Preparation of study reports

\section{Project Director Assistant: Vu Thi Tuong Vi}

Overseeing the study implementation in Ho Chi Minh City

\section{Ethnographer: Dinh Thi Thanh Thuy, Nguyen Hong Hoa}

Development of qualitative data collection tools

Conducting in-depth interviews, focus group discussion and field observation

Intervention Coaches and Master Behavioural Counselors: Han Dinh Hoe, Nguyen Huu Anh, Nguyen Ly Lai, Pham Thi Dan Linh

Providing training and technical assistance to practitioners at methadone clinics who deliver the study interventions

Providing the SMS messaging intervention

\section{References}

1. Ellis MS, Kasper ZA, Cicero TJ. Twin epidemics: The surging rise of methamphetamine use in chronic opioid users. Drug Alcohol Depend. 2018 Dec 1;193:14-20.

2. Giang L, Thuy D, Trang N, Okafor C, Li M, Shoptaw S. Severity of methamphetamine use among methadone patients in Vietnam: Prevalence and correlates. In San Antonio, Texas; 2019.

3. Michel L, Des Jarlais DC, Duong Thi H, Khuat Thi Hai O, Pham Minh K, Peries M, et al. Intravenous heroin use in Haiphong, Vietnam: Need for comprehensive care including methamphetamine use-related interventions. Drug Alcohol Depend. 2017 Oct;179:198-204. 
4. UNODC GS programme. Synthetic Drugs in East and Southeast Asia - Latest developments and challenges. 2020 May p. 116.

5. Bao Y-P, Liu Z-M, Li J-H, Zhang R-M, Hao W, Zhao M, et al. Club drug use and associated high-risk sexual behaviour in six provinces in China. Addict Abingdon Engl. 2015 Jan;110 Suppl 1:11-9.

6. Carrico AW, Nil E, Sophal C, Stein E, Sokunny M, Yuthea N, et al. Behavioral interventions for Cambodian female entertainment and sex workers who use amphetamine-type stimulants. J Behav Med. 2016;39(3):502-10.

7. Colfax G, Santos G-M, Chu P, Vittinghoff E, Pluddemann A, Kumar S, et al. Amphetamine-group substances and HIV. Lancet Lond Engl. 2010 Aug 7;376(9739):458-74.

8. Zhang C, Liu Y, Sun X, Wang J, Lu H-Y, He X, et al. Substance use and HIV-risk behaviors among HIVpositive men who have sex with men in China: repeated measures in a cohort study design. AIDS Care. 2017;29(5):644-53.

9. Liu D, Gu J, Xu H, Hao C, Jiao M, Zhang X, et al. Club drugs and alcohol abuse predicted dropout and poor adherence among methadone maintenance treatment patients in Guangzhou, China. AIDS Care. 2017;29(4):458-63.

10. Wang R, Ding Y, Bai H, Duan S, Ye R, Yang Y, et al. Illicit Heroin and Methamphetamine Use among Methadone Maintenance Treatment Patients in Dehong Prefecture of Yunnan Province, China. PLOS ONE. 2015 Jul 21;10(7):e0133431.

11. Carrico AW, Cherenack EM, Roach ME, Riley ED, Oni O, Dilworth SE, et al. Substance-associated elevations in monocyte activation among methamphetamine users with treated HIV infection. AIDS Lond Engl. 2018 27;32(6):767-71.

12. Carrico AW, Hunt PW, Neilands TB, Dilworth SE, Martin JN, Deeks SG, et al. Stimulant Use and Viral Suppression in the Era of Universal Antiretroviral Therapy. JAIDS J Acquir Immune Defic Syndr. 2019 Jan 1;80(1):89-93.

13. Carrico AW, Flentje A, Kober K, Lee S, Hunt P, Riley ED, et al. Recent stimulant use and leukocyte gene expression in methamphetamine users with treated HIV infection. Brain Behav Immun. 2018;71:108-15.

14. Horvath KJ, Carrico AW, Simoni J, Boyer EW, Amico KR, Petroll AE. Engagement in HIV Medical Care and Technology Use among Stimulant-Using and Nonstimulant-Using Men who have Sex with Men. AIDS Res Treat [Internet]. 2013 [cited 2021 Jan 25];2013. Available from:

https://www.ncbi.nlm.nih.gov/pmc/articles/PMC3705882/

15. Korthuis, P. Todd, Bart, Gavin, Kunkel L, Diep NB, Brinkerhoff C, Giang LM. Buprenorphine versus Methadone impact on the HIV care continuum in Vietnam: BRAVO study 6-month outcomes. In San Diego; 2018. 
16. Alam-mehrjerdi Z, Mokri A, Dolan K. Methamphetamine use and treatment in Iran: A systematic review from the most populated Persian Gulf country. Asian J Psychiatry. 2015 Aug;16:17-25.

17. Shariatirad S, Maarefvand M, Ekhtiari H. Methamphetamine use and methadone maintenance treatment: an emerging problem in the drug addiction treatment network in Iran. Int J Drug Policy. 2013 Nov;24(6):e115-116.

18. Watt MH, Meade CS, Kimani S, MacFarlane JC, Choi KW, Skinner D, et al. The impact of methamphetamine ("tik") on a peri-urban community in Cape Town, South Africa. Int J Drug Policy. 2014 Mar;25(2):219-25.

19. Baingana F, al'Absi $M$, Becker $A E$, Pringle B. Global research challenges and opportunities for mental health and substance-use disorders. Nature. 2015 Nov 19;527(7578):S172-177.

20. Petersen I, Evans-Lacko S, Semrau M, Barry MM, Chisholm D, Gronholm P, et al. Promotion, prevention and protection: interventions at the population- and community-levels for mental, neurological and substance use disorders in low- and middle-income countries. Int J Ment Health Syst. 2016;10:30.

21. AshaRani P, Hombali A, Seow E, Ong WJ, Tan JH, Subramaniam M. Non-pharmacological interventions for methamphetamine use disorder: a systematic review. Drug Alcohol Depend. $2020 \mathrm{Jul}$ 1;212:108060.

22. Knight R, Karamouzian M, Carson A, Edward J, Carrieri P, Shoveller J, et al. Interventions to address substance use and sexual risk among gay, bisexual and other men who have sex with men who use methamphetamine: A systematic review. Drug Alcohol Depend. 2019 Jan;194:410-29.

23. Radfar SR, Rawson RA. Current Research on Methamphetamine: Epidemiology, Medical and Psychiatric Effects, Treatment, and Harm Reduction Efforts. Addict Health. 2014;6(3-4):146-54.

24. Polcin DL, Bond J, Korcha R, Nayak MB, Galloway GP, Evans K. Randomized Trial of Intensive Motivational Interviewing for Methamphetamine Dependence. J Addict Dis. 2014 Jul 3;33(3):253-65.

25. Galloway GP, Polcin D, Kielstein A, Brown M, Mendelson J. A Nine Session Manual of Motivational Enhancement Therapy for Methamphetamine Dependence: Adherence and Efficacy. J Psychoactive Drugs. 2007 Nov 1;39(sup4):393-400.

26. Benishek LA, Dugosh KL, Kirby KC, Matejkowski J, Clements NT, Seymour BL, et al. Prize-based contingency management for the treatment of substance abusers: a meta-analysis. Addict Abingdon Engl. 2014 Sep;109(9):1426-36.

27. Brown HD, DeFulio A. Contingency management for the treatment of methamphetamine use disorder: A systematic review. Drug Alcohol Depend. 2020 Nov;216:108307. 
28. Dutra L, Stathopoulou G, Basden SL, Leyro TM, Powers MB, Otto MW. A meta-analytic review of psychosocial interventions for substance use disorders. Am J Psychiatry. 2008 Feb;165(2):179-87.

29. Griffith JD, Rowan-Szal GA, Roark RR, Simpson DD. Contingency management in outpatient methadone treatment: a meta-analysis. Drug Alcohol Depend. 2000 Feb 1;58(1-2):55-66.

30. Prendergast M, Podus D, Finney J, Greenwell L, Roll J. Contingency management for treatment of substance use disorders: a meta-analysis [Internet]. Centre for Reviews and Dissemination (UK); 2006 [cited 2020 Feb 25]. Available from: https://www.ncbi.nlm.nih.gov/books/NBK72392/

31. Lussier JP, Heil SH, Mongeon JA, Badger GJ, Higgins ST. A meta-analysis of voucher-based reinforcement therapy for substance use disorders. Addict Abingdon Engl. 2006 Feb;101(2):192-203.

32. Ginley MK, Pfund RA, Rash CJ, Zajac K. Long-term efficacy of contingency management treatment based on objective indicators of abstinence from illicit substance use up to 1 year following treatment: $A$ meta-analysis. J Consult Clin Psychol. 2021 Jan;89(1):58-71.

33. Rawson RA, Marinelli-Casey P, Anglin MD, Dickow A, Frazier Y, Gallagher C, et al. A multi-site comparison of psychosocial approaches for the treatment of methamphetamine dependence. Addict Abingdon Engl. 2004 Jun;99(6):708-17.

34. Reback CJ, Fletcher JB, Shoptaw S, Mansergh G. Exposure to Theory-Driven Text Messages is Associated with HIV Risk Reduction Among Methamphetamine-Using Men Who have Sex with Men. AIDS Behav. 2015 Jun;19 Suppl 2:130-41.

35. Reback CJ, Fletcher JB, Swendeman DA, Metzner M. Theory-based Text-Messaging to Reduce Methamphetamine Use and HIV Sexual Risk Behaviors among Men who have Sex with Men: Automated Unidirectional Delivery Outperforms Bidirectional Peer Interactive Delivery. AIDS Behav. 2019 Jan;23(1):37-47.

36. Reback CJ, Fletcher JB, Kisler KA. Text Messaging Improves HIV Care Continuum Outcomes Among Young Adult Trans Women Living with HIV: Text Me, Girl! AIDS Behav. 2021 Sep;25(9):3011-23.

37. Reback CJ, Fletcher JB, Leibowitz AA. Cost effectiveness of text messages to reduce methamphetamine use and HIV sexual risk behaviors among men who have sex with men. J Subst Abuse Treat. 2019 May;100:59-63.

38. Gómez W, Olem D, Andrews R, Discepola MV, Ambrose P, Dilworth SE, et al. Optimizing Contingency Management With Methamphetamine-Using Men Who Have Sex With Men. Cogn Behav Pract. 2018 May 1;25(2):286-95.

39. Parsons JT, John SA, Millar BM, Starks TJ. Testing the Efficacy of Combined Motivational Interviewing and Cognitive Behavioral Skills Training to Reduce Methamphetamine Use and Improve HIV 
Medication Adherence Among HIV-Positive Gay and Bisexual Men. AIDS Behav. 2018 Aug;22(8):267486.

40. Dugosh K, Abraham A, Seymour B, McLoyd K, Chalk M, Festinger D. A Systematic Review on the Use of Psychosocial Interventions in Conjunction With Medications for the Treatment of Opioid Addiction. J Addict Med. 2016 Mar;10(2):91-101.

41. Assanangkornchai S, Balthip Q, Edwards JG, assistance of the ASSIST-SBI Development Coinvestigators. Implementing the Alcohol, Smoking, Substance Involvement Screening Test and linked brief intervention service in primary care in Thailand. J Public Health Oxf Engl. 2014 Sep;36(3):443-9.

42. Sorsdahl K, Myers B, Ward C, Matzopoulos R, Mtukushe B, Nicol A, et al. Screening and brief interventions for substance use in emergency departments in the Western Cape province of South Africa: views of health care professionals. Int J Inj Contr Saf Promot. 2014;21(3):236-43.

43. Wechsberg WM, Luseno WK, Karg RS, Young S, Rodman N, Myers B, et al. Alcohol, cannabis, and methamphetamine use and other risk behaviors among Black and Coloured South African women: a small randomized trial in the Western Cape. Int J Drug Policy. 2008 Apr;19(2):130-9.

44. Chen W, Hong Y, Zou X, McLaughlin MM, Xia Y, Ling L. Effectiveness of prize-based contingency management in a methadone maintenance program in China. Drug Alcohol Depend. 2013 Nov 1;133(1):270-4.

45. Hser Y-I, Li J, Jiang H, Zhang R, Du J, Zhang C, et al. Effects of a randomized contingency management intervention on opiate abstinence and retention in methadone maintenance treatment in China. Addict Abingdon Engl. 2011 Oct;106(10):1801-9.

46. Cohen A, McGregor C. The Emergence of Methamphetamine in Thailand: Interventions and Treatment. In Richard Pates, Diane Riley. In: Interventions for Amphetamine Misuse. Oxford: WileyBlackwell Publishing. 2010. p. 159-71.

47. Magidson JF, Gouse H, Burnhams W, Wu CYY, Myers B, Joska JA, et al. Beyond methamphetamine: Documenting the implementation of the Matrix model of substance use treatment for opioid users in a South African setting. Addict Behav. 2017;66:132-7.

48. Nguyen Huu C, Kieu Cong Thuy. Application of Matrix model in support of trreatment and recovery for ATS dependents (Nghiên cứu sử dụng liệu pháp tâm lý Matrix trong hỗ trợ điều trị nghiện và phục hồi chức năng cho người nghiện ma túy tổng hợp dạng amphetamine). In Hanoi, Vietnam; 2016.

49. Curran GM, Bauer M, Mittman B, Pyne JM, Stetler C. Effectiveness-implementation Hybrid Designs. Med Care. 2012 Mar;50(3):217-26.

50. Damschroder LJ, Aron DC, Keith RE, Kirsh SR, Alexander JA, Lowery JC. Fostering implementation of health services research findings into practice: a consolidated framework for advancing implementation 
science. Implement Sci. 2009 Aug 7;4(1):50.

51. Damschroder LJ, Hagedorn HJ. A guiding framework and approach for implementation research in substance use disorders treatment. Psychol Addict Behav. 2011;25(2):194-205.

52. Hanoi Centre for Disease Control. MMT program data. 2021.

53. HCMC Centre for Disease Control. MMT program data. 2021.

54. Roll JM, Shoptaw S. Contingency management: Schedule effects. Psychiatry Res. 2006 Sep 30;144(1):91-3.

55. UNAIDS. Costing Guidelines for HIV Prevention Strategies. 2000.

56. Hintz J. PASS 12 [Internet]. 2013 [cited 2021 Apr 23]. Available from: https://www.ncss.com/

57. Lei H, Nahum-Shani I, Lynch K, Oslin D, Murphy SA. A "SMART" design for building individualized treatment sequences. Annu Rev Clin Psychol. 2012;8:21-48.

58. Almirall D, Nahum-Shani I, Sherwood NE, Murphy SA. Introduction to SMART designs for the development of adaptive interventions: with application to weight loss research. Transl Behav Med. 2014 Sep;4(3):260-74.

59. Nahum-Shani I, Qian M, Almirall D, Pelham WE, Gnagy B, Fabiano GA, et al. Experimental design and primary data analysis methods for comparing adaptive interventions. Psychol Methods. 2012 Dec;17(4):457-77.

60. Neumann PJ, Sanders GD, Russell LB, Ganiats TG, Siegel JE. Cost-effectiveness in Health and Medicine. Oxford University Press; 2017. 537 p.

\section{Tables}

Table 1. Assessment schedule 


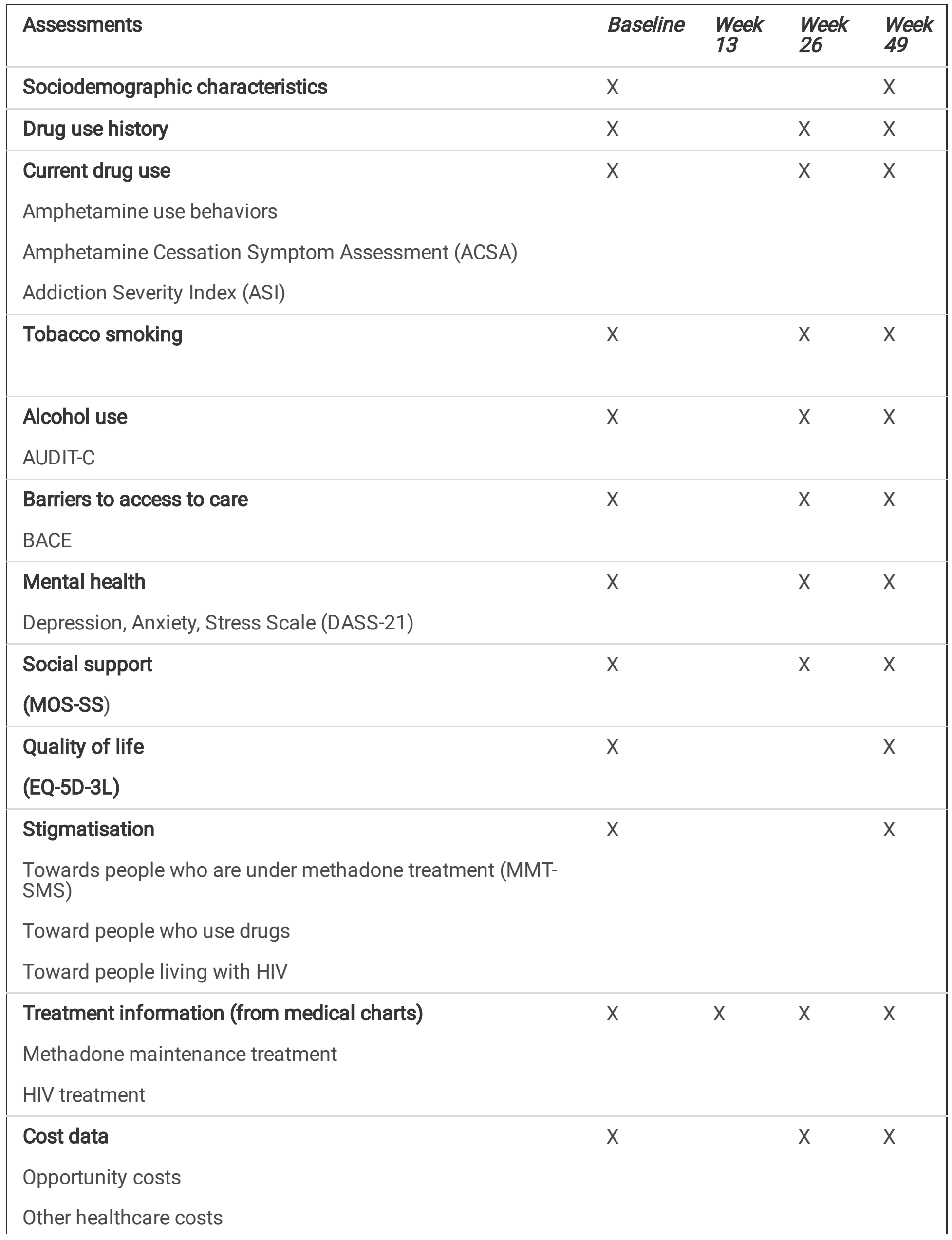




\section{Tests}

Quick and confirmatory HIV tests (not for participants with known HIV seropositivity)

Viral load test

UDS

Ethnographic observation

Qualitative interviews

$X \quad X \quad X$

Twice a week throughout $\quad \mathrm{X}$ intervention

$\begin{array}{llll}X & X & X & X\end{array}$

$x \quad x \quad x \quad x$

$\mathrm{X} \quad \mathrm{X}$

Table 2. Number of eligible participants by original criteria

\begin{tabular}{|llll|}
\hline & \# eligible (ASSIST+UDS) & UDS (+) & ASSIST $\geq 4$ \\
\hline Hanoi clinic $(\mathrm{N}=350)$ & 26 & 31 & 114 \\
\hline HCMC clinic $(\mathrm{N}=385)$ & 52 & 64 & 122 \\
\hline
\end{tabular}

\section{Figures}

\section{Figure 1}

\section{Study flowchart}

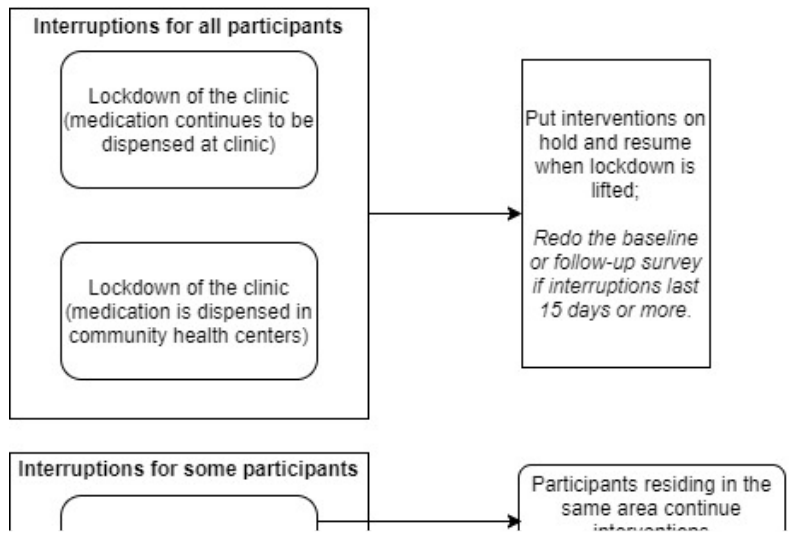

Notes:

- Missed group work will be substituted by individual

sessions of similar content if needed.

- No missing data due to COVID-19

Decision criteria on the continuation of intervention 
Figure 2

Response plan to COVID-19 related interruptions

\section{Supplementary Files}

This is a list of supplementary files associated with this preprint. Click to download.

- SPIRITchecklist.docx 